\title{
Spontaneous strain buffer enables superior cycling stability in single-crystal nickel-rich NCM cathode
}

He Zhu ${ }^{a \dagger}$, Yu Tang ${ }^{a \dagger}$, Kamila M. Wiaderek ${ }^{b}$, Olaf J. Borkiewicz, ${ }^{b}$ Yang Ren ${ }^{a}$, Jian Zhang ${ }^{a}$, Jincan Ren ${ }^{a}$, Longlong $\mathrm{Fan}^{b}$, Cheng Chao $\mathrm{Li}^{c}$, Danfeng $\mathrm{Li}^{a}$, Xun-Li Wang ${ }^{a, d}$, Qi Liu ${ }^{a, d^{*}}$

${ }^{a}$ Department of Physics and Center for Neutron Scattering, City University of Hong Kong, Hong Kong 999077, P. R. China

${ }^{b}$ College of Physics and Materials Science, Tianjin Normal University, Tianjin 300387, P. R. China

${ }^{c}$ School of Chemical Engineering and Light Industry, Guangdong University of Technology, Guangzhou 510006, P. R. China

${ }^{d}$ Shenzhen Research Institute, City University of Hong Kong, Shenzhen 518057, P. R. China

Corresponding to: qiliu63@cityu.edu.hk

†These authors contributed equally: He Zhu and Yu Tang. 


\section{Experimental Section}

Synthesis of the SC- and PC-N83 cathode materials

Spherical $\left[\mathrm{Ni}_{0.83} \mathrm{Co}_{0.12} \mathrm{Mn}_{0.05}\right](\mathrm{OH})_{2}$ precursors were synthesized using a typical coprecipitation method. An aqueous solution containing $\mathrm{NiSO}_{4} \cdot 6 \mathrm{H}_{2} \mathrm{O}, \mathrm{CoSO}_{4} \cdot 7 \mathrm{H}_{2} \mathrm{O}$, and $\mathrm{MnSO}_{4} \cdot 5 \mathrm{H}_{2} \mathrm{O}$ with a concentration of $2.0 \mathrm{~mol} \mathrm{~L}-1$ was pumped into a continuously stirring tank reactor under $\mathrm{N}_{2}$ atmosphere. At the same time, $\mathrm{NaOH}$ solution with a concentration of $4.0 \mathrm{~mol} \mathrm{~L}^{-1}$ and a desired amount $\mathrm{NH}_{4} \mathrm{OH}$ solution were pumped separately into the reactor to maintain $\mathrm{pH} \sim 11$ during the reaction. Afterwards, the precursors were filtered, thoroughly washed with distilled water, and dried overnight at $110^{\circ} \mathrm{C}$

The SC-N83 cathode material was prepared by using a facile calcination-grinding method on the co-precipitated precursors. In detail, $\mathrm{LiOH}$ was thoroughly mixed with the precursors with a molar ratio of 1.05: 1 . The excess lithium in the raw material was used for the compensation of lithium loss during the calcination process. After that, a two-stage calcination process was carried out — the mixture was preliminarily annealed at $500{ }^{\circ} \mathrm{C}$ for $5 \mathrm{~h}$, and subsequently calcined at $880^{\circ} \mathrm{C}$ for $10 \mathrm{~h}$ in oxygen atmosphere. The obtained cathode material was grounded by ball milling for $4 \mathrm{~h}$ with a speed of 300 $\mathrm{rpm} \mathrm{min}^{-1}$. The grounded material was calcinated again at $750{ }^{\circ} \mathrm{C}$ for $10 \mathrm{~h}$ and the SCN83 was prepared. By comparison, the PC-N83 cathode material was prepared by mixing $\mathrm{LiOH}$ and precursors with a molar ratio of $1.02: 1$, and then the mixture was calcinated at $800{ }^{\circ} \mathrm{C}$ for $10 \mathrm{~h}$. 


\section{Electrochemical measurements}

The PC-N83 and SC-N83 electrodes were prepared by mixing $80 \mathrm{wt} . \%$ active materials, $10 \mathrm{wt} . \%$ super $\mathrm{P}$ and $10 \mathrm{wt} \%$ poly (vinylidene fluoride) (PVDF) uniformly in the solvent of N-methyl-2-pyrrolidone. The uniform slurry was placed on Al foil current collector before dried at $90{ }^{\circ} \mathrm{C}$ in vacuum for $12 \mathrm{~h}$. The mass loading of the active material per electrode was about $4 \mathrm{mg} \mathrm{cm}^{-2}$. Then the electrodes were assembled to 2032 coin-type cells in an Argon-filled glovebox using lithium metal as the counter electrode. The electrolyte used in the coin cells was $1.2 \mathrm{M} \mathrm{LiPF}_{6}$ in ethylene carbonateethyl methyl carbonate $(3: 7$, vol \%) with 2 wt \% vinylene carbonate (VC). The cells were charged and discharged between $2.8 \mathrm{~V}$ and $4.3 \mathrm{~V}$ at different rates $(1 \mathrm{C}=190$ $\mathrm{mAh} \mathrm{g}^{-1}$ ) on NEWARE Battery test system at room temperature. The EIS tests were conducted by using an amplitude of $5 \mathrm{mV}$ in the frequency range of $0.01 \mathrm{~Hz}$ and 1 MHz. The temperature of the EIS testing was controlled by Ivium-n-Stat multichannel electrochemical analyser. The EIS plots are fitted according to the equivalent circuit. The first circle in the high frequency is related to the resistance for the migration of lithium ions through the surface film $\left(R_{\mathrm{f}}\right)$, while the second one in the low frequency is attributed to the charge transfer resistance $\left(R_{\mathrm{ct}}\right)$. The temperature-dependent $R_{\mathrm{ct}}$ follows the Arrhenius equation described as:

$$
\frac{1}{R_{c t}}=A \exp \left(-\frac{E_{a}}{R T}\right)
$$

where $\mathrm{A}$ is the frequency factor, $E_{\mathrm{a}}$ is the activation energy, $R$ is the gas constant, and $T$ is the absolute temperature. The GITT measurements were conducted to evaluate the 
$\mathrm{Li}^{+}$diffusion coefficients $\left(D_{L i}{ }^{+}\right)$based on the Fick's second law of diffusion. The equation for $D_{L i}{ }^{+}$calculation can be written as below:

$$
D_{L i^{+}}=\frac{4}{\pi \tau}\left(\frac{m_{B} V_{M}}{M_{B} A}\right)^{2}\left(\frac{\Delta E_{S}}{\Delta E_{\tau}}\right)^{2}
$$

Where $m_{\mathrm{B}}, V_{\mathrm{M}}, M_{\mathrm{B}}$ and $A$ are the mass, the molar volume, the molecular weight, and the interface area of the electrode material, respectively. The calculation of $\Delta E_{\mathrm{s}}$ and $\Delta E_{\tau}$ is shown in Figure $\mathbf{S 4 a}$.

\section{Sample characterizations}

The powder XRD measurements were performed using a Smart Lab diffractometer (Rigaku Corporation, Tokyo, Japan), equipped with a $\mathrm{Cu} K \alpha$ radiation $(\lambda=1.54186 \AA)$ and a HyPix-3000 detector. The collected XRD patterns were refined based on the Rietveld method using Fullprof software. The backgrounds were subtracted by linear interpolation and the peak shapes were described using a pseudo-Voigt profile function. The SEM images were taken by JSM-7800F field emission scanning electron microscope (FESEM) operated at an acceleration voltage of $20 \mathrm{kV}$, while the TEM and HRTEM images were taken on JEOL JEM-2100 equipment operated at $200 \mathrm{keV}$. For the in situ lab XRD test, a specially designed cell with Al window and Al current collector was used for the first cycle. To ensure the high-rate measurement, every scan was collected in $0.02^{\circ}$ increments between $10^{\circ}$ and $55^{\circ}$ at a scanning speed of $0.18^{\circ}$ per second.

Collection and analysis of in situ synchrotron XRD 
The in situ XRD experiments were carried out using the 11-ID-C beamline at Advanced Photon Source (APS) of Argonne National Laboratory (ANL), with the X-ray wavelength $0.1173 \AA$. Si (113) single crystal was employed as monochromator for an X-ray beam at $105.7 \mathrm{keV}$. The in situ XRD data was collected using Argonne's multipurpose in situ X-ray (AMPIX) cell. ${ }^{[1]}$ The cathode pellet was prepared by mixing the SC-N83 powders with super-P carbon and PVDF binder uniformly in weight ratio of 6:2:2 in $\sim 2 \mathrm{~mL}$ of N-methyl-2-pyrrolidone, and this pellet was mounted in the AMPIX cell with Li metal as anode. The electrolyte used in the coin cells was $1.2 \mathrm{M} \mathrm{LiPF}_{6}$ in ethylene carbonate-ethyl methyl carbonate (3:7, vol \%) with 2 wt \% vinylene carbonate (VC). More detailed introduction, schematic diagram, and photograph of the AMPIX cell could be found in Ref [1]. In a typical in situ collection, a single XRD pattern was obtained in the transmission geometry with a Perkin-Elmer two-dimensional detector during a time period of 30 seconds. The obtained $2 \mathrm{D}$ patterns were calibrated with a standard sample of $\mathrm{CeO}_{2}$, and then integrated into 1D patterns using the Fit2D software. The lattice parameters were extracted from the integrated XRD patterns using Fullprof software.

\section{Collection and analysis of in situ PDF of synchrotron X-ray total scattering}

The AMPIX cell was used for the in situ PDF collection with the fabrication as described above. The collection of raw scattering data was similar to that of in situ XRD, except that the sample-to-detector distance is closer for a high value of momentum transfer $\left(Q_{\max } \sim 20.6 \AA\right)$. The collected 2D scattering images were reduced to onedimensional data using Fit2D software. The resulted one-dimensional data was 
corrected with PDFgetX3 software for background and Campton scatterings, and to compute the $\mathrm{G}(r)$ functions, $G(r)=4 \pi r\left[\rho(r)-\rho_{\mathrm{o}}\right]$, where $\rho(r)$ and $\rho_{\mathrm{o}}$ are the local and average atomic number densities, by Fourier transform. ${ }^{[2]}$ The PDF refinements were carried out against the relevant structure models using PDFgui software. ${ }^{[3]}$ 


\section{Supplementary Figures}
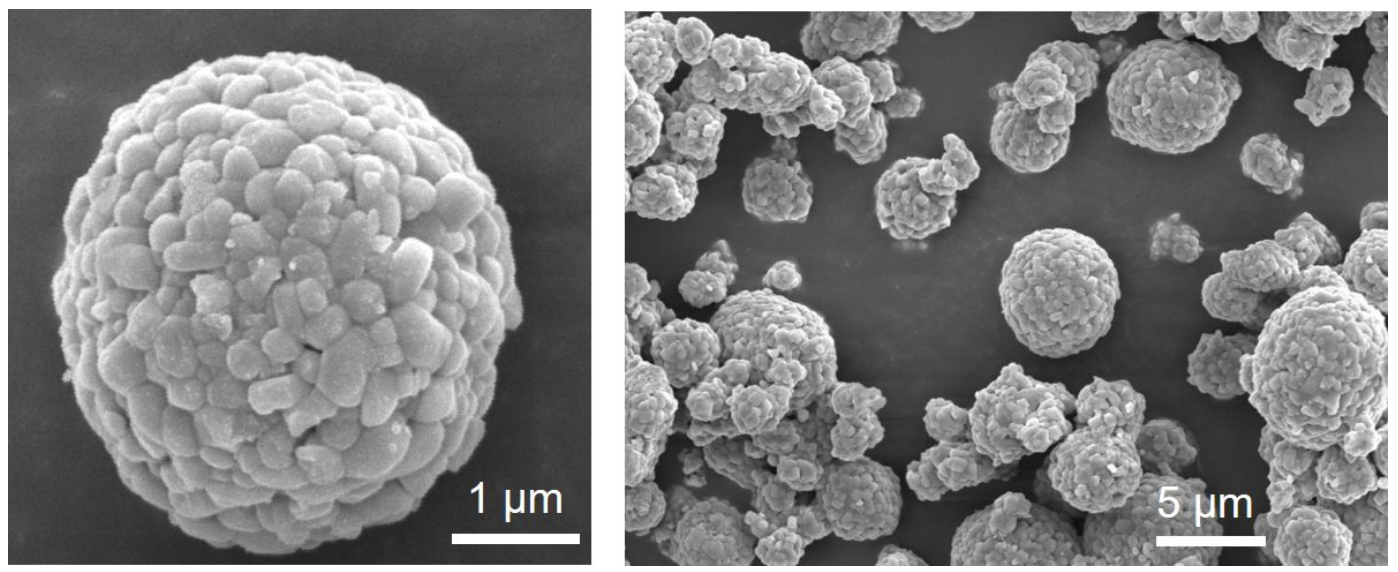

Figure S1. Morphology of the as-prepared PC-N83 particles characterized by SEM. 

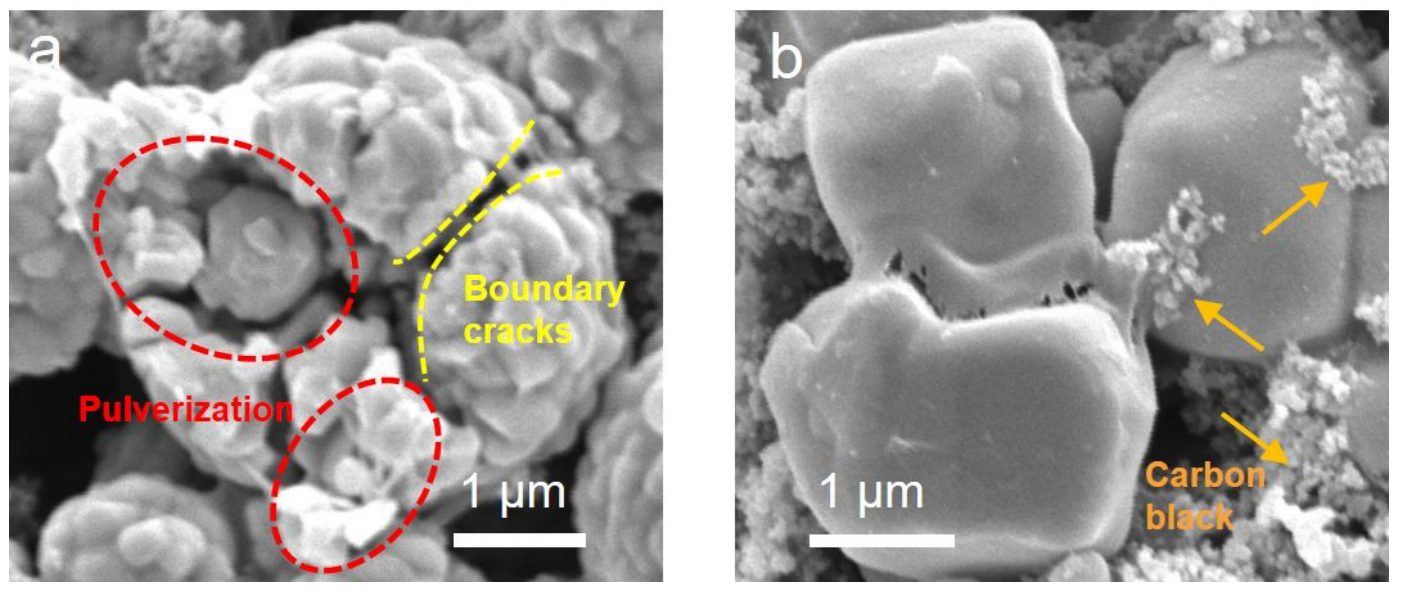

Figure S2. SEM images of a) PC-N83 and b) SC-N83 electrode particles after 200 cycles. 

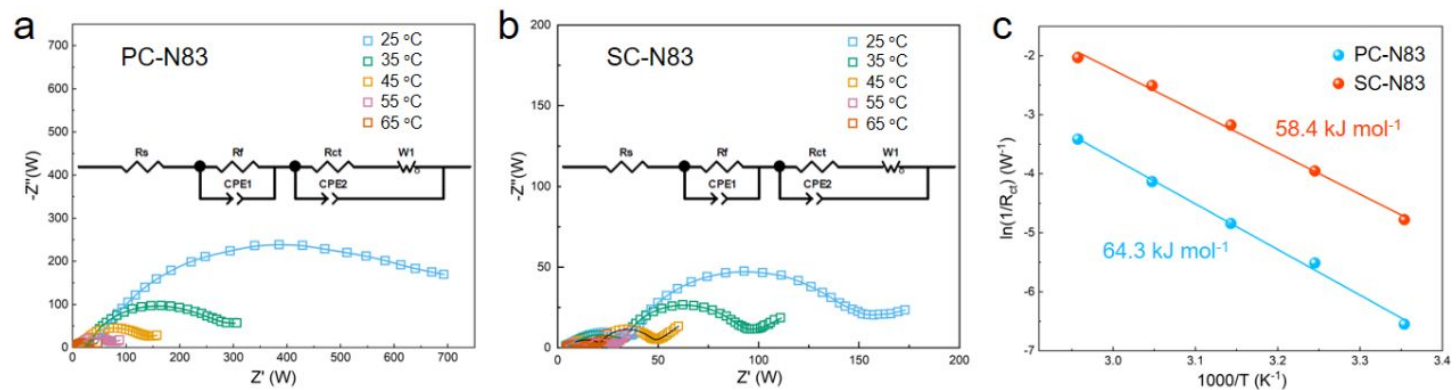

Figure S3. a) EIS plots of the PC-N83 and b) SC-N83 samples after running 200 cycles at variable temperatures. The insets are the equivalent circuit models to describe the EIS plots. c) Temperature dependencies of $R_{\mathrm{ct}}$ for the PC- and SC-N83 electrodes after 200 cycles. 

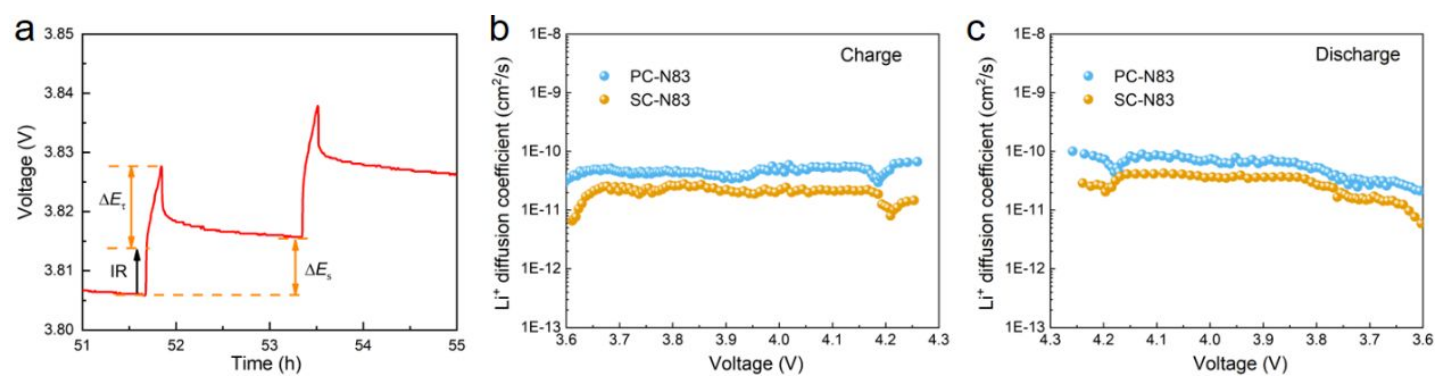

Figure S4. a) Amplified voltage response within one single current pulse. Calculated $\mathrm{Li}^{+}$diffusion coefficients of PC- and SC-N83 cathodes at b) charge and c) discharge process. 


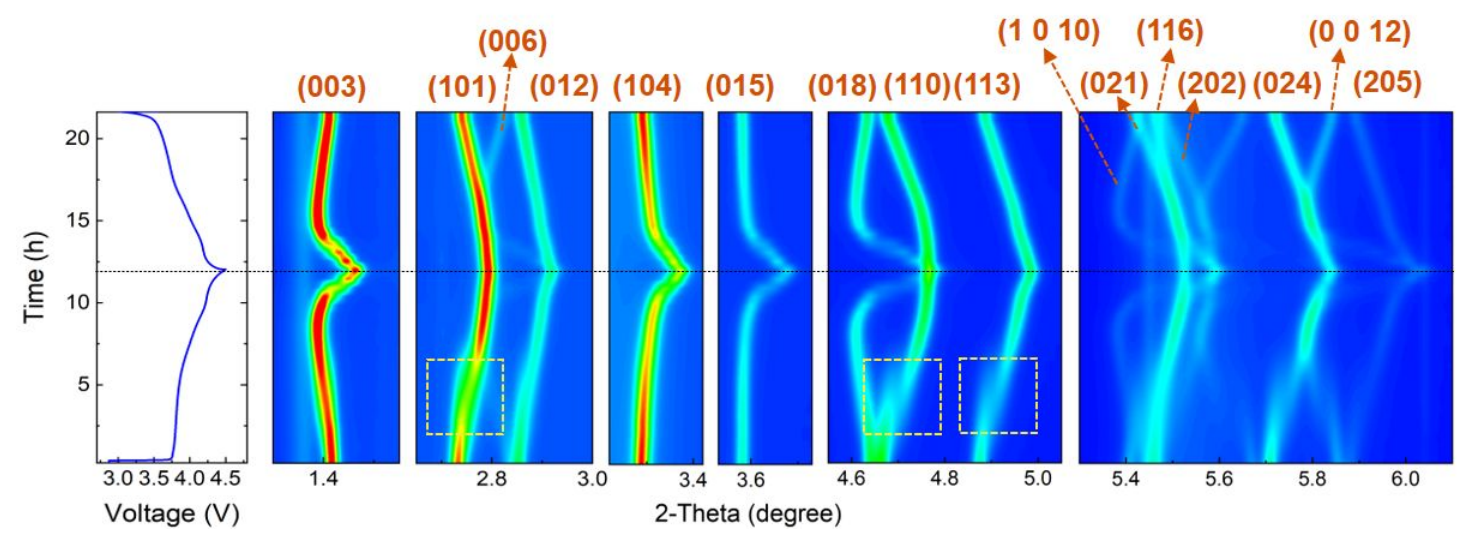

Figure S5. Contour plots of in situ XRD pattern for the SC-N83 cathode coupled with the voltage profile. The yellow dotted boxes frame the peak splits that fingerprint the monoclinic phase. 

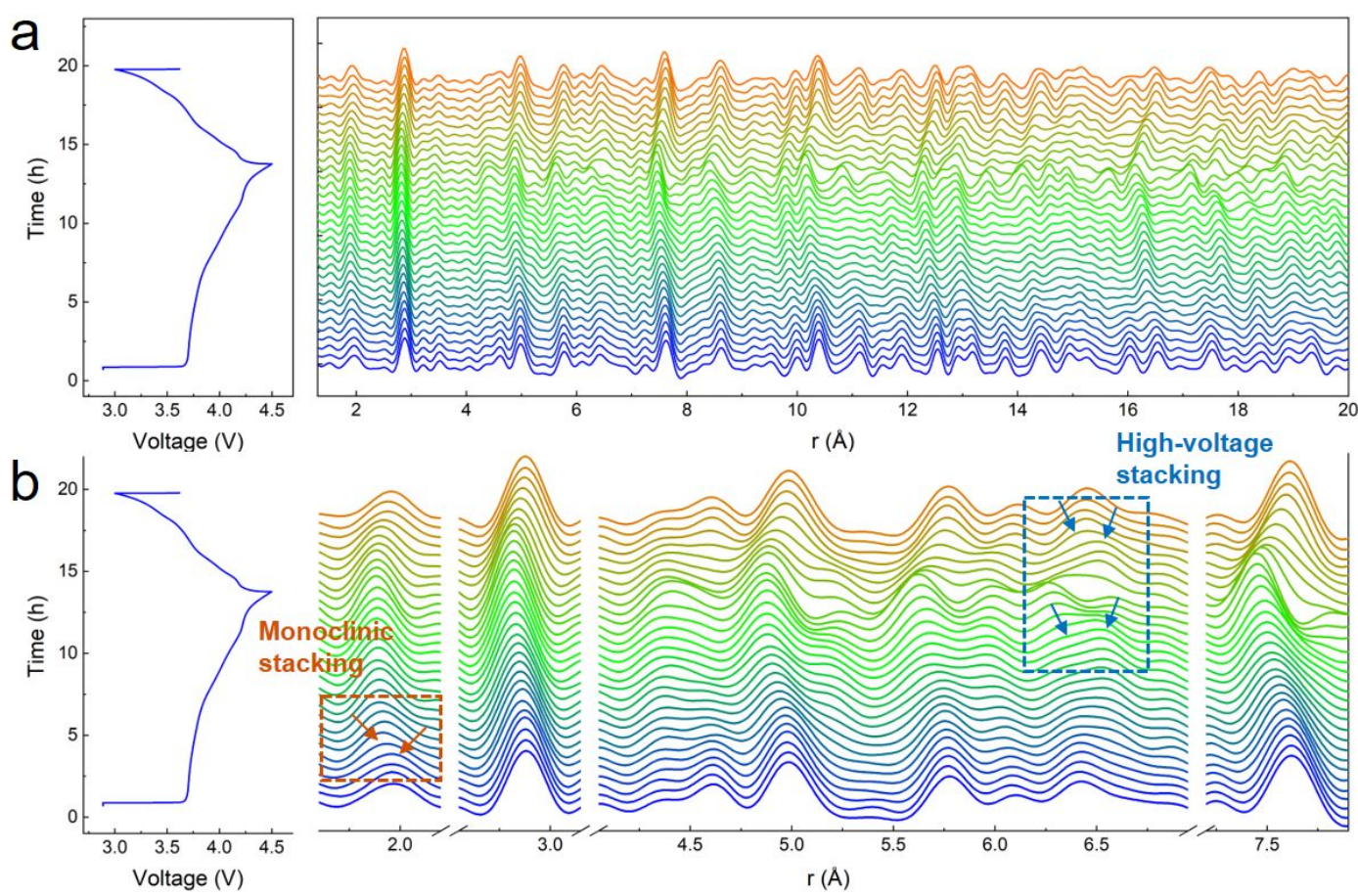

Figure S6. a) Stacked plots of in situ PDF patterns for SC-N83 cathode coupled with the corresponding voltage profile within one charge-discharge cycle. b) Stacked profiles of low- $r$ PDF peaks within the initial charge-discharge process. The red box and arrows show the first-shell split of the monoclinic phase, while the blue box and arrows indicate the high-voltage stacking faults across the interlayers. 

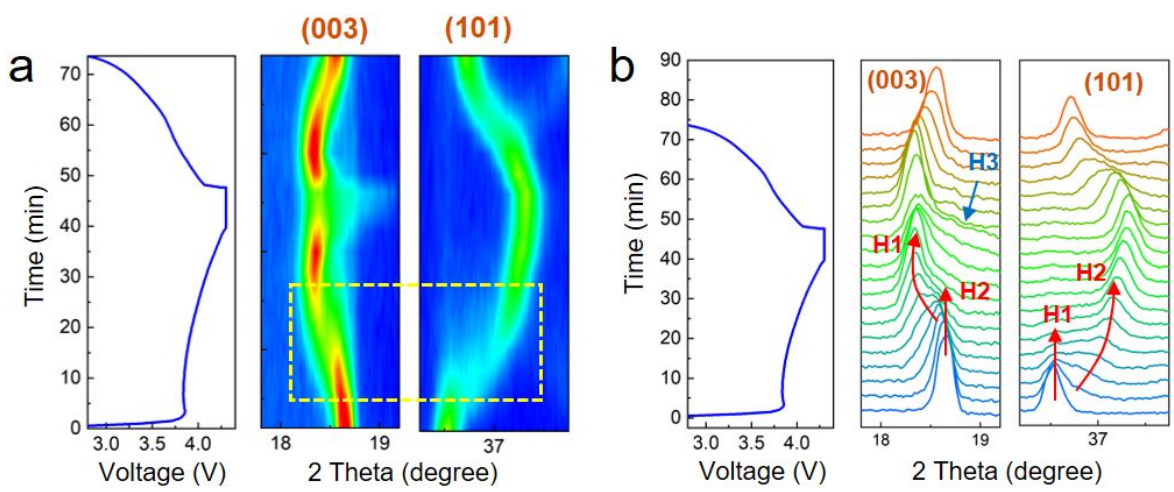

Figure S7. a) Contour plot and b) stacked plot of in situ XRD measurement with the corresponding charge-discharge profile at a high rate of $2 \mathrm{C}$ on the $\mathrm{SC}-\mathrm{N} 83$ cathode. The yellow dashed box in a) emphasizes the coexistance region of $\mathrm{H} 1$ and $\mathrm{H} 2$ upon charging. 


\section{Supplementary Tables}

Table S1. Lattice constants and $I_{003} / I_{104}$ ratios obtained from XRD Rietveld analysis based on the $R-3 m$ space group.

\begin{tabular}{ccccccc}
\hline Samples & a-axis $(\AA)$ & $c$-axis $(\AA)$ & $c / a$ & $V\left(\AA^{3}\right)$ & $I_{003} / I_{104}$ & $R_{\text {wp }}$ \\
\hline SC-N83 & $2.87605(1)$ & $14.19623(4)$ & $4.9383(1)$ & $101.742(1)$ & 1.577 & 4.21 \\
PC-N83 & $2.87477(1)$ & $14.18983(6)$ & $4.9387(1)$ & $101.614(1)$ & 1.498 & 4.48 \\
\hline
\end{tabular}

Table S2. Lattice parameters of SC-N83 extracted from full-profile PDF refinement based on $R-3 m$ space group.

\begin{tabular}{ccccc}
\hline Atom & $x$ & $y$ & $z$ & Occ. \\
\hline $\mathrm{Li}$ & 0 & 0 & 0.5 & 1.0 \\
$\mathrm{Ni}$ & 0 & 0 & 0 & 0.83 \\
$\mathrm{Co}$ & 0 & 0 & 0 & 0.12 \\
$\mathrm{Mn}$ & 0 & 0 & 0 & 0.05 \\
$\mathrm{O}$ & 0 & 0 & $0.259(1)$ & 1 \\
\hline \multicolumn{5}{l}{} \\
\hline
\end{tabular}




\section{Reference}

[1] J. Borkiewicz, B. Shyam, K. M. Wiaderek, C. Kurtz, P. J. Chupas, K. W. Chapman, J. Appl. Crystallogr. 45 (2012) 1261-1269.

[2] P. Juhás, T. Davis, C. L. Farrow, S. J. Billinge, J. Appl. Crystallogr. 46 (2013) $560-566$.

[3] C. Farrow, P. Juhas, J. Liu, D. Bryndin, E. Božin, J. Bloch, T. Proffen, S. Billinge, J. Phys. Condens. Matter. 19 (2007) 335219. 